\title{
The effect of figure on syllogistic reasoning
}

\author{
LOUIS S. DICKSTEIN \\ Wellesley College, Wellesley, Massachusetts 02181
}

\begin{abstract}
Several investigators of reasoning with abstract categorical syllogisms have noted that the four figures of the classical syllogism, which vary in the order in which terms occur in the major and minor premises, resemble the four three-stage mediation paradigms in paired associate learning. On the basis of the analogy, figure differences have been predicted and some support has been obtained for these predictions. The present paper proposes an alternative information processing explanation for figure effects based upon contradictions between the correct conclusions that follow from forward and backward processing of the premises. This explanation, in contrast to the associative explanation, successfully predicts which premise combinations will show figure effects as well as the nature of the specific errors which will occur.
\end{abstract}

In recent years, several investigators have concerned themselves with the cognitive processes involved in performance on abstract categorical syllogisms (e.g., Dickstein, 1975, 1976; Erickson, 1974; Revlis, 1975a, 1975b). The general intent of these studies has been to clarify the operations that subjects perform in interpreting premises, combining information and drawing conclusions. While the focus of these studies has been the categorical syllogism, the more general aim has been to identify processes which might have generality beyond the specific deductive reasoning task. This paper continues the effort to articulate the psychological bases of performance on logical tasks.

Much empirical research on syllogistic reasoning has been concerned with factors that contribute to differences in difficulty among the various syllogisms. For example, it has been found that valid syllogisms are considerably easier than invalid syllogisms (Dickstein, 1975, 1976; Roberge, 1970). Within the class of invalid syllogisms, syllogisms that are susceptible to the logical errors of illicit conversion of universal affirmative or particular negative propositions are more difficult than syllogisms that do not allow such errors (Chapman \& Chapman, 1959; Dickstein, 1975, 1976). This paper is concerned with the role of the figure of the syllogism as a determinant of difficulty.

A categorical syllogism consists of two premises followed by a conclusion. Both premises and the conclusion make assertions about class relationships between various terms. Traditionally, the first premise is the major premise, which makes an assertion about the relation between the middle term $(M)$ and the predicate (P) (e.g., All $\mathrm{M}$ are $\mathrm{P}$ ). The second premise

The main ideas in this paper were developed while the author was on sabbatical leave from Wellesley College at the Institute for the Study of Intellectual Behavior, University of Colorado. The leave was partially funded by Postdoctoral Research Fellowship 1 F 32 MH05214-01 to the author from the National Institute of Mental Health under the sponsorship of Lyle E. Bourne, Jr. is the minor premise, which makes an assertion about the relation between the subject (S) and M (e.g., All S are $M$ ). On the basis of the information provided by the two premises, the task of the subject is to draw a deductive inference about the relation between $S$ and $P$. For a valid syllogism, the conclusion would be a propositional statement about the class relationship between $\mathbf{S}$ and $\mathbf{P}$ (e.g., All $S$ are P). For an invalid syllogism, the correct conclusion would be that no valid propositional conclusion may be drawn about the relationship between $\mathrm{S}$ and $\mathrm{P}$.

In the syllogistic task, there are four kinds of propositions that are traditionally represented by letters. These are universal affirmative (A) propositions (e.g., All $M$ are $P$ ), universal negative (E) propositions (e.g., No $M$ are $P$ ), particular affirmative (I) propositions (e.g., Some $M$ are P), and particular negative $(0)$ propositions (e.g., Some $M$ are not $P$ ). Since there are four different kinds of propositional statements that can occur in both the major and minor premises, there are 16 different premise combinations in the syllogistic task.

Syllogisms may also vary with regard to the ordering of the terms in the major and minor premises. Thus, the major premise may be stated with $M$ preceding $P$, or it may be stated with $P$ preceding $M$. Similarly, the minor premise may be stated with $S$ preceding $M$, or it may be stated with $\mathrm{M}$ preceding $\mathrm{S}$. The order of the terms in the major and minor premises defines the figure of the syllogism. In Figure 1 the order of the terms for the major and minor premises, respectively, is $\mathrm{M}-\mathrm{P}, \mathrm{S}-\mathrm{M}$; in Figure 2 the order is P-M, S-M; in Figure 3 the order is M-P, M-S; while in Figure 4 the order is P-M, M-S. The conclusion to be evaluated in all instances proceeds from $S$ to $P$. Since there are four figures with 16 different premise combinations in each figure, the complete syllogistic task consists of 64 unique syllogisms. The various kinds of propositions and the various premise orders are summarized in Table 1. 
Table 1

Types of Propositions and Orders of Terms for Categorical Syllogisms

Types of Propositions

$\begin{array}{lc}\text { A } & \text { All } S \text { are } P \\ \mathrm{E} & \text { No S are } \mathrm{P} \\ \mathrm{I} & \text { Some } \mathrm{S} \text { are } \mathrm{P} \\ \mathrm{O} & \text { Some } S \text { are not } \mathrm{P} \\ & \text { Orders of Terms }\end{array}$

$\begin{array}{cccc}\text { Figure 1 } & \text { Figure 2 } & \text { Figure 3 } & \text { Figure 4 } \\ \text { M-P } & \text { P-M } & \text { M-P } & \text { P-M } \\ \text { S-M } & \text { S-M } & \text { M-S } & \text { M-S }\end{array}$

The order of the terms in the premises significantly affects the meaning of some propositions ( $A$ and $O$ ). Thus, the proposition "All M are P" does not have the same logical meaning as the proposition "All $\mathrm{P}$ are M." Consequently, a premise combination that yields a valid conclusion in one figure may yield a different valid conclusion or may be indeterminate in a different figure. Of the 64 syllogisms that constitute the task, 19 are valid and the remaining 45 are invalid. There are four valid syllogisms in Figure 1, four in Figure 2, six in Figure 3, and five in Figure 4.

Several investigators beginning with Frase (1968) have noted that the four figures of the syllogistic task are analogous to the four three-stage mediation paradigms in paired associate learning. Figures 1 , 2, 3, and 4 resemble forward chaining, stimulus equivalence, response equivalence, and backward chaining, respectively. On the basis of this analogy, it has been argued that performance should become progressively poorer as one proceeds from Figure 1 to Figure 4 , and some evidence has been advanced in support of this prediction. Thus, in the first of two experiments reported by Frase (1968), the difference between figures approached significance, with subjects making least errors on Figure 1 syllogisms and most errors on Figure 4 syllogisms. In the second experiment, the difference between figures was significant, with errors increasing from Figure 1 to Figure 4. These experiments, however, used a very limited sample of the total set of categorical syllogisms. A subsequent study by Roberge (1971) compared figures for the nine different premise combinations that have the same logically correct conclusion in all four figures. For these 36 syllogisms, he found that Figure 4 was significantly more difficult than Figures 2 and 3 . More recently, Erickson (1974) has presented data for the 19 valid syllogisms in which the percentages correct are $79 \%$ for Figure 1, 76\% for Figure 2, 59\% for Figure 3, and $49 \%$ for Figure 4. Although Erickson's (1974) model of syllogistic reasoning is not based upon the mediation paradigm analogy, he does note that these results parallel those from verbal mediation studies. Further confirmation of the difficulty of Figure 4 syllogisms has been reported as an incidental finding by Kintsch and Monk (1972) in a study of memory that included meaningful materials presented in a syllogistic structure.

Although the analogy between paired associates and syllogisms is interesting, it is difficult to regard the mediation analogy as an adequate explanation for figure differences. Over the years, a number of theoretical explanations of reasoning on categorical syllogisms have been advanced, including those of Chapman and Chapman (1959), Erickson (1974), Revlis (1975a, 1975b), and Woodworth and Sells (1935). A distinguishing feature of all of these approaches has been the presentation of some form of processing model leading to the prediction of specific errors for particular premise combinations. In contrast, the mediation explanation for figure effects does not provide a processing model, nor does it predict the specific errors that will occur in figures that are more difficult. Since it is well established that errors on this task are not random (e.g., Dickstein, 1975), the mediation analogy appears to be seriously incomplete.

The thesis of the present paper is that it is more productive to view syllogistic reasoning as an information processing activity, in which the subject interprets the premises and combines the information in the premises to arrive at a conclusion, than to view subject performance in terms of an associative model. Such an analysis suggests an alternative explanation for figure effects. As noted above, in the traditional syllogism task, propositional conclusions proceed from $S$ to $P$, and the subject must decide whether such conclusions are valid or whether no valid propositional conclusion may be deduced which proceeds from $\mathrm{S}$ to $\mathrm{P}$. However, just as the information in the premises can be processed to evaluate conclusions proceeding from $S$ to $P$, the same information can be processed to evaluate conclusions proceeding from $P$ to $S$. The logically correct conclusion regarding the relation from $S$ to $P$ is not necessarily correct with regard to the relation from $P$ to $S$. It is proposed that figure effects occur on those syllogisms in which conclusions that are logically justified from $P$ to $S$ are different from conclusions that are justified from $S$ to $P$. Even though the task requires subjects to draw conclusions that are appropriate from $S$ to $P$, some subjects will draw conclusions that are appropriate from $P$ to $S$ and then erroneously apply them to the relation from $S$ to $P$. It is further proposed that whether the subject processes the information in the premises in a forward (subject to predicate) or backward (predicate to subject) direction will be influenced by the direction of the information presented in the premises. In Figure 1, both premises proceed in the forward direction, and there should be little tendency for subjects to process information from $P$ to $S$. In contrast, in Figure 4 both premises proceed from $P$ to $S$ and here the tendency to process information in the backward direction should 
be maximal. Figures 2 and 3 are intermediate since, in both, one premise proceeds in a forward direction, while one proceeds in a backward direction. Thus, it is hypothesized that for those premise combinations in which forward and backward processing lead to different conclusions, performance will be best on Figure 1 syllogisms, intermediate on Figures 2 and 3 , and poorest on Figure 4. Furthermore, this decrement across figures in the number of correct conclusions will be paralleled by an increase in the number of errors resulting from backward processing.

Among the 64 syllogisms, there are 18 in which different conclusions are justified from $P$ to $S$ than from $S$ to $P$. These include eight syllogisms that allow propositional conclusions from $S$ to $\mathbf{P}$ but are invalid from $\mathrm{P}$ to $\mathrm{S}$. These are syllogisms EI-1, EI-2, EI-3, EI-4, EA-3, EA-4, A0-2, and OA-3. (In these abbreviations, the first letter refers to the kind of proposition in the major premise, the second letter refers to the kind of proposition in the minor premise, and the number refers to the figure of the syllogism.) There are also eight syllogisms that are invalid from $\mathrm{S}$ to $\mathrm{P}$ but allow propositional conclusions from $\mathbf{P}$ to $\mathbf{S}$. These are syllogisms IE-1, IE-2, IE-3, IE-4, AE-1, AE-3, OA-2, and AO-3. Finally, there are two syllogisms that lead to different propositional conclusions from $\mathrm{S}$ to $\mathrm{P}$ than from $P$ to $S$. These are AA-1 and AA-4.

Unfortunately, the main hypothesis of the present study cannot be tested with all 18 relevant syllogisms. In order to attribute differences between syllogisms to the effect of figure, the syllogisms to be compared must be equated as to premise combination, logically correct conclusion, and susceptibility to errors that have been identified in previous research, such as illicit conversion (Chapman \& Chapman, 1959). Otherwise, the effect of figure is confounded with the effects of other variables. This caution has not always been observed in previous research. Thus, Erickson (1974) compared figures with regard to valid syllogisms, even though different premise combinations are represented in the different figures. More seriously, the figure comparison which Erickson (1974) presents is confounded with the effects of illicit conversion. There are only four valid syllogisms in which illicit conversion leads to error (AA-3, AA-4, EA-3, and EA-4) and these are the most difficult valid syllogisms. Since conversion has been shown to be a very strong error tendency (Chapman \& Chapman, 1959; Dickstein, 1975, 1976), it is more parsimonious to attribute the poorer performance of subjects on Figures 3 and 4 to conversion than to a vaguely defined figure effect. Furthermore, since there are six valid syllogisms in Figure 3 and only five in Figure 4, the difficult conversion syllogisms constitute a larger proportion of the total set for Figure 4, and this can account for the difference between Figures 3 and 4 reported by Erickson (1974).
After consideration of these requirements, there are 12 syllogisms in four premise combinations for which figure differences may be predicted and tested. Premise combination EI in all four figures leads to a valid $\mathrm{O}$ conclusion in the forward direction and a nonpropositional conclusion in the backward direction. Conversely, premise combination IE in all four figures leads to a nonpropositional conclusion in the forward direction and an $\mathrm{O}$ conclusion in the backward direction. Premise combination EA in Figures 3 and 4 leads to a valid $O$ conclusion in the forward direction and a nonpropositional conclusion in the backward direction. Finally, premise combination $\mathrm{AE}$ in Figures 1 and 3 leads to a nonpropositional conclusion in the forward direction and an $\mathrm{O}$ conclusion in the backward direction.

The present study also provides an opportunity to test the proposal of Revlis (1975b) that subjects have a response bias against nonpropositional conclusions. Premise combinations EI and IE are complementary syllogisms. In both there is one universal negative proposition and one particular affirmative proposition. However, in the former, forward processing leads to a propositional $\mathrm{O}$ conclusion and backward processing leads to a nonpropositional conclusion, while in the latter the reverse is true. If subjects have a bias against nonpropositional conclusions, then performance should be better on the valid EI syllogisms than on the invalid IE syllogisms.

\section{METHOD}

Two samples of subjects provided the data of the present study. The first consisted of 22 female undergraduates at Wellesley College who had participated in an earlier study of syllogistic reasoning (Dickstein, 1975) as the standard instruction group. These subjects completed 64 syllogisms including all four figures. Half of the subjects received the syllogisms in the standard order with the major premise first, while the other half received the reverse order. Since the two orders of presentation were not significantly different, the data were combined.

The second sample consisted of 76 female undergraduates at Wellesley College who participated in the study to meet a course requirement. These subjects were randomly divided into four groups of 19. Each group completed all the syllogisms for a single figure, with two presentations of each syllogism, for a total of 32 syllogisms. There were no significant differences between the first and second presentations, and the data were combined.

All subjects received problem booklets with instructions that clarified the rules of the syllogistic task. Subjects were told that the word "some" means "at least some" and not "only some." In addition, subjects were told that in order to draw a substantive conclusion, the premises had to compel that conclusion. Each premise combination was followed by five response alternatives, and the subject was required to select one of them. The alternatives were: "All S are P," "Some S are $P$," "No $S$ are $P$," "Some $S$ are not $P$," and "No valid conclusion." These alternatives were always presented in the same order to avoid confusing the subjects. Similarly, the letters $S, M$, and $P$ were always used to represent the subject, middle term, and predicate to avoid confusion. Subjects completed 
all of the syllogisms at a single session and no time limit was imposed.

\section{RESULTS}

The mean number correct and the mean predicted error for each of the 12 syllogisms for Sample 1 and Sample 2 are presented in Table 2. Since premise combinations EI and IE are represented in all four figures, analyses of variance were conducted with these eight syllogisms to test the effects of figure, validity, and any interaction between them.

For the first sample, where a within-subjects design was utilized, two treatments by treatments by subjects analyses of variance (Bruning \& Kintz, 1968) were conducted. One analysis utilized the number of correct responses as the dependent variable, while the other utilized the number of the predicted error responses as the dependent variable. ${ }^{1}$ For the correct response, there was a significant effect of figure $[F(3,63)=3.76$, $\mathrm{p}<.05$ ], with performance on Figure 1 best, performance on Figures 2 and 3 combined intermediate, and performance on Figure 4 poorest. There was also a significant effect of validity $[F(1,21)=5.86 . p<.05]$, with performance on valid premise combination EI significantly better than performance on invalid premise combination IF. The interaction between figure and validity was not significant $[F(3,63)=2.62]$. For the predicted error, there was again a significant effect of figure $[F(3,63)=3.07, p<.05]$, with the least error in Figure 1, an intermediate amount of error in Figures 2 and 3 combined, and the greatest amount of error in Figure 4 . The effects of validity and the Figure by Validity interaction were not significant.

For the second sample, where validity was a withinsubjects factor while figure was a between-subjects

Table 2

Mean Percentage Occurrence of Predicted Error and Correct Answer

\begin{tabular}{crrrrr}
\hline & \multicolumn{2}{c}{ Sample 1 } & & \multicolumn{2}{c}{ Sample 2 } \\
\cline { 2 - 3 } Combination & Error & Correct & & Error & Correct \\
\hline EA-3 & 0.0 & 18.2 & & 2.6 & 23.7 \\
EA-4 & 18.2 & 27.3 & & 34.2 & 5.3 \\
EI-1 & 18.2 & 68.2 & 7.9 & 68.4 \\
E1-2 & 13.6 & 72.7 & & 21.1 & 57.9 \\
EI-3 & 27.3 & 54.5 & & 18.4 & 55.3 \\
EI-4 & 36.4 & 40.9 & & 50.0 & 23.7 \\
AE-1 & 0.0 & 50.0 & & 5.3 & 36.8 \\
AE-3 & 18.2 & 18.2 & & 7.9 & 18.4 \\
IE-1 & 9.1 & 59.1 & & 13.2 & 68.4 \\
IE-2 & 45.5 & 31.8 & & 36.8 & 18.4 \\
IE-3 & 40.9 & 22.7 & & 21.1 & 39.5 \\
IE-4 & 36.4 & 36.4 & 34.2 & 34.2 \\
\hline
\end{tabular}

Note-The predicted error is "no valid conclusion" for premise combinations $E A$ and $E I$ and " $O$ " for premise combinations $A E$ and $I E$. The correct answer is " $O$ " for premise combinations $E A$ and $E I$ and "no valid conclusion" for premise combinations $A E$ and $I E$. factor, a two-factor mixed-design (Bruning \& Kintz, 1968) analysis of variance was utilized. Again, two analyses were conducted for the number correct and for the predicted error. For the correct response, there was a significant effect of figure $[F(3,72)=5.86$, $\mathrm{p}<.01$ ], with performance on Figure 1 best, performance on Figures 2 and 3 combined intermediate, and performance on Figure 4 poorest. The effect of validity was not significant $[F(1,72)=3.58]$. However, there was a significant Figure by Validity interaction $[F(3,72)=3.40, \quad p<.05]$, reflecting the relatively poor performance of subjects on Figure 4 compared to the other figures for premise combination EI as compared with the relatively good performance on Figure 1 compared to the other figures for premise combination IE. For the predicted error, there was again a significant effect of figure $[F(3,72)=6.00$, $\mathrm{p}<.01]$, with the least error in Figure 1, an intermediate amount of error in Figures 2 and 3 combined, and the greatest amount of error in Figure 4. The effects of validity and the Figure by Validity interaction were not significant.

For premise combinations $\mathrm{EA}$ and $\mathrm{AE}$, in which syllogisms from only two figures were represented, $t$ tests were conducted for both samples on both the number of correct responses and the number of predicted error responses. For the first sample, for premise combination $\mathrm{EA}$, the difference between Figure 3 and Figure 4 was not significant for the number correct but was significant for the predicted error $[\mathrm{t}(21)=2.17, \mathrm{p}<.05$, two-tailed $]$, with more predicted errors occurring in Figure 4. For premise combination $\mathrm{AE}$, the difference between Figure 1 and Figure 3 was significant for the number correct $[\mathrm{t}(21)=2.63$, $p<.02$, two-tailed], with more correct responses in Figure 1. There was also a significant difference for the predicted error $[\mathrm{t}(21)=2.17, \mathrm{p}<.05$, twotailed], with more predicted errors occurring in Figure 3.

For the second sample, for premise combination EA, the difference between Figure 3 and Figure 4 approached significance for the number correct $[\mathrm{t}(36)=1.79, \mathrm{p}<.10$, two-tailed $]$, with more correct responses in Figure 3. For the predicted error, the difference was significant $[\mathrm{t}(36)=3.00, \mathrm{p}<.01$, two-tailed], with more predicted errors in Figure 4. For premise combination $\mathrm{AE}$, the difference between Figures 1 and 3 was not significant for either the number correct or the predicted error.

In addition to the comparisons presented above in which figure differences are predicted, there are a number of other comparisons in the total set of syllogisms for which the model predicts that there will not be figure differences because the conclusion to be drawn is the same for both forward processing and backward processing. These comparisons involve 13 different premise combinations and 44 individual syllogisms. 
The largest subset of these syllogisms consists of seven premise combinations that are invalid in all four figures, both in terms of forward processing and backward processing. These are premise combinations EE, EO, OE, OO, II, IO, and OI. For the first sample, a treatments by subjects analysis of variance was conducted based upon the number correct over the seven syllogisms in each figure. Figure differences were not significant $[F(3,63)=1.98]$. For the second sample, an analysis of variance for a randomized design was conducted and, again, figure effects were not significant $[F(3,72)=.64]$.

In addition to these 28 syllogisms, there are eight other comparisons of pairs of syllogisms in which the conclusions to be drawn from both forward and backward processing are the same. These include four pairs in which the same valid propositional conclusion is justified from both forward and backward processing (AE-2 and $\mathrm{AE}-4$, AI- 1 and AI-3, IA-3 and IA-4, and EA-1 and EA-2) and four invalid pairs in which the correct answer from both forward and backward processing is that no propositional conclusion may be drawn (AI-2 and AI-4, IA-1 and IA-2, $\mathrm{AO}-1$ and $\mathrm{AO} 4$, and $\mathrm{OA}-1$ and $\mathrm{OA}-4$ ). The se comparisons were analyzed with $t$ tests for related measures for the first sample and with $t$ tests for independent groups for the second sample. As predicted by the model, none of the $16 \mathrm{t}$ tests for the two samples was significant. The highest $t$ value occurred for the comparison of syllogisms $\mathrm{AO}-1$ and $\mathrm{AO}-4$ in the first sample, and this t reached only 1.70 . The median t for the 16 separate comparisons was only .76 . Thus, there were no unpredicted figure effects.

In addition to tests of the particular hypotheses of the study, analyses of variance were conducted to test for the figure effect, based on all 19 valid syllogisms reported by Erickson (1974). For the first sample, performance decreased from Figure 1 to Figure 4, with $90.9 \%, 88.6 \%, 62.9 \%$, and $53.6 \%$ correct, respectively. This figure effect was highly significant $[F(3,63)=32.52, p<.001]$. In the second sample, performance also decreased from Figure 1 to Figure 4, with $86.2 \%, 79.6 \%, 60.1 \%$, and $45.8 \%$ correct, respectively. Again, the effect of figure was highly significant $[F(3,72)=17.30, p<.001]$.

Finally, since there do not appear to be any presentations of the complete response distributions for all 64 syllogisms in the literature, these data are presented here in their entirety. Table 3 presents the data for the first sample, in which the same subjects received all 64 syllogisms, and Table 4 presents the data for the second sample in which each figure was administered to a different set of subjects.

\section{DISCUSSION}

The results of the present study indicate that figure is a significant determinant of performance within a specific subset of syllogisms. Figure effects on the
Table 3

Response Distributions for 64 Syllogisms, Sample 1

\begin{tabular}{|c|c|c|c|c|c|}
\hline Syllogism & A & $\mathrm{E}$ & 1 & $\mathrm{O}$ & $\mathrm{N}^{*}$ \\
\hline AA-1 & 95.5 & 0.0 & 0.0 & 0.0 & 4.5 \\
\hline $\mathrm{AA}-2$ & 77.3 & 0.0 & 0.0 & 0.0 & 22.7 \\
\hline AA-3 & 59.1 & 0.0 & 27.3 & 0.0 & 13.6 \\
\hline $\mathrm{AA} 4$ & 59.1 & 0.0 & 22.7 & 4.5 & 13.6 \\
\hline $\mathrm{AE}-1$ & 0.0 & 50.0 & 0.0 & 0.0 & 50.0 \\
\hline AE-2 & 0.0 & 95.5 & 0.0 & 0.0 & 4.5 \\
\hline AE-3 & 0.0 & 63.6 & 0.0 & 18.2 & 18.2 \\
\hline $\mathrm{AE}-4$ & 0.0 & 86.4 & 0.0 & 4.5 & 9.1 \\
\hline Al-1 & 0.0 & 0.0 & 100.0 & 0.0 & 0.0 \\
\hline AI-2 & 0.0 & 0.0 & 81.8 & 4.5 & 13.6 \\
\hline AI-3 & 0.0 & 0.0 & 90.9 & 0.0 & 9.1 \\
\hline AI-4 & 0.0 & 0.0 & 68.2 & 9.1 & 22.7 \\
\hline AO-1 & 0.0 & 0.0 & 0.0 & 86.4 & 13.6 \\
\hline $\mathrm{AO}-2$ & 0.0 & 0.0 & 0.0 & 90.9 & 9.1 \\
\hline AO- 3 & 0.0 & 0.0 & 4.5 & 63.6 & 31.8 \\
\hline $\mathrm{AO}-4$ & 0.0 & 0.0 & 0.0 & 68.2 & 31.8 \\
\hline EA-1 & 0.0 & 100.0 & 0.0 & 0.0 & 0.0 \\
\hline EA-2 & 0.0 & 95.5 & 0.0 & 4.5 & 0.0 \\
\hline EA-3 & 0.0 & 81.8 & 0.0 & 18.2 & 0.0 \\
\hline EA-4 & 0.0 & 54.5 & 0.0 & 27.3 & 18.2 \\
\hline EE-1 & 0.0 & 27.3 & 0.0 & 0.0 & 72.7 \\
\hline EE-2 & 0.0 & 27.3 & 0.0 & 4.5 & 68.2 \\
\hline EE-3 & 0.0 & 22.7 & 0.0 & 0.0 & 77.3 \\
\hline $\mathrm{EE}-4$ & 0.0 & 18.2 & 0.0 & 4.5 & 77.3 \\
\hline EI-1 & 0.0 & 13.6 & 0.0 & 68.2 & 18.2 \\
\hline EI-2 & 0.0 & 13.6 & 0.0 & 72.7 & 13.6 \\
\hline EI-3 & 0.0 & 18.2 & 0.0 & 54.5 & 27.3 \\
\hline EI-4 & 0.0 & 22.7 & 0.0 & 40.9 & 36.4 \\
\hline EO-1 & 0.0 & 18.2 & 4.5 & 22.7 & 54.5 \\
\hline EO-2 & 0.0 & 9.1 & 13.6 & 4.5 & 72.7 \\
\hline $\mathrm{EO}-3$ & 0.0 & 9.1 & 0.0 & 4.5 & 86.4 \\
\hline $\mathrm{EO}-4$ & 0.0 & 18.2 & 0.0 & 4.5 & 77.3 \\
\hline IA-1 & 0.0 & 0.0 & 72.7 & 0.0 & 27.3 \\
\hline IA-2 & 4.5 & 0.0 & 63.6 & 4.5 & 27.3 \\
\hline IA-3 & 4.5 & 4.5 & 90.9 & 0.0 & 0.0 \\
\hline IA-4 & 0.0 & 0.0 & 90.9 & 4.5 & 4.5 \\
\hline IE-1 & 0.0 & 31.8 & 0.0 & 9.1 & 59.1 \\
\hline IE-2 & 0.0 & 22.7 & 0.0 & 45.5 & 31.8 \\
\hline IE-3 & 0.0 & 36.4 & 0.0 & 40.9 & 22.7 \\
\hline IE-4 & 0.0 & 22.7 & 4.5 & 36.4 & 36.4 \\
\hline II-1 & 0.0 & 0.0 & 50.0 & 4.5 & 45.5 \\
\hline II-2 & 0.0 & 4.5 & 63.4 & 0.0 & 31.8 \\
\hline II-3 & 0.0 & 0.0 & 36.4 & 0.0 & 63.6 \\
\hline II-4 & 0.0 & 0.0 & 40.9 & 0.0 & 59.1 \\
\hline IO-1 & 0.0 & 0.0 & 4.5 & 40.9 & 54.5 \\
\hline IO-2 & 0.0 & 0.0 & 0.0 & 59.1 & 40.9 \\
\hline IO-3 & 0.0 & 0.0 & 0.0 & 31.8 & 68.2 \\
\hline IO-4 & 0.0 & 0.0 & 0.0 & 40.9 & 59.1 \\
\hline OA-1 & 0.0 & 0.0 & 0.0 & 77.3 & 22.7 \\
\hline $\mathrm{OA}-2$ & 0.0 & 9.1 & 0.0 & 68.2 & 22.7 \\
\hline $\mathrm{OA}-3$ & 0.0 & 0.0 & 0.0 & 95.5 & 4.5 \\
\hline $\mathrm{OA}-4$ & 0.0 & 0.0 & 0.0 & 63.6 & 36.4 \\
\hline OE-1 & 0.0 & 18.2 & 0.0 & 0.0 & 81.8 \\
\hline OE-2 & 0.0 & 13.6 & 13.6 & 0.0 & 72.7 \\
\hline $\mathrm{OE}-3$ & 0.0 & 18.2 & 9.1 & 4.5 & 68.2 \\
\hline $\mathrm{OE} 4$ & 0.0 & 9.1 & 4.5 & 18.2 & 68.2 \\
\hline OI-1 & 0.0 & 0.0 & 4.5 & 40.9 & 54.5 \\
\hline Ol-2 & 0.0 & 0.0 & 0.0 & 36.4 & 63.6 \\
\hline OI-3 & 0.0 & 0.0 & 0.0 & 50.0 & 50.0 \\
\hline OI-4 & 0.0 & 4.5 & 0.0 & 31.8 & 63.6 \\
\hline OO-1 & 0.0 & 0.0 & 0.0 & 27.3 & 72.7 \\
\hline OO-2 & 0.0 & 0.0 & 9.1 & 13.6 & 77.3 \\
\hline OO-3 & 0.0 & 0.0 & 9.1 & 13.6 & 77.3 \\
\hline $\mathrm{OO}-4$ & 0.0 & 4.5 & 4.5 & 9.1 & 81.8 \\
\hline
\end{tabular}

Note-Numbers indicate percentage of total responses to each syllogism. $\quad$ *N refers to "no valid conclusion." 
Table 4

Response Distributions for 64 Syllogisms, Sample 2

\begin{tabular}{|c|c|c|c|c|c|}
\hline Syllogism & A & $\mathrm{E}$ & I & $\mathrm{O}$ & $\mathrm{N}^{*}$ \\
\hline AA-1 & 94.7 & 0.0 & 2.6 & 0.0 & 2.6 \\
\hline AA-2 & 68.4 & 2.6 & 5.3 & 2.6 & 21.1 \\
\hline AA-3 & 65.8 & 0.0 & 28.9 & 0.0 & 5.3 \\
\hline AA 4 & 71.1 & 2.6 & 21.1 & 0.0 & 5.3 \\
\hline AE-1 & 0.0 & 52.6 & 5.3 & 5.3 & 36.8 \\
\hline $\mathrm{AE}-2$ & 0.0 & 94.7 & 0.0 & 2.6 & 2.6 \\
\hline$A E-3$ & 0.0 & 71.1 & 2.6 & 7.9 & 18.4 \\
\hline $\mathrm{AE}-4$ & 0.0 & 89.5 & 5.3 & 2.6 & 2.6 \\
\hline AI-1 & 0.0 & 5.3 & 89.5 & 5.3 & 0.0 \\
\hline Al-2 & 0.0 & 5.3 & 63.2 & 21.1 & 10.5 \\
\hline Al-3 & 2.6 & 2.6 & 89.5 & 5.3 & 0.0 \\
\hline $\mathrm{Al}-4$ & 0.0 & 0.0 & 76.3 & 0.0 & 23.7 \\
\hline $\mathrm{AO}-1$ & 2.6 & 2.6 & 2.6 & 55.3 & 36.8 \\
\hline $\mathrm{AO}-2$ & 0.0 & 5.3 & 7.9 & 73.7 & 13.2 \\
\hline $\mathrm{AO}-3$ & 0.0 & 0.0 & 15.8 & 65.8 & 18.4 \\
\hline $\mathrm{AO}-4$ & 0.0 & 5.3 & 5.3 & 73.7 & 15.8 \\
\hline EA-1 & 0.0 & 92.1 & 2.6 & 5.3 & 0.0 \\
\hline EA-2 & 0.0 & 92.1 & 0.0 & 5.3 & 2.6 \\
\hline EA-3 & 0.0 & 73.7 & 0.0 & 23.7 & 2.6 \\
\hline $\mathrm{EA}_{-4}$ & 2.6 & 52.6 & 5.3 & 5.3 & 34.2 \\
\hline EE-1 & 0.0 & 34.2 & 2.6 & 2.6 & 60.5 \\
\hline $\mathrm{EE}-2$ & 5.3 & 13.2 & 5.3 & 5.3 & 71.1 \\
\hline $\mathrm{EE}-3$ & 0.0 & 18.4 & 0.0 & 0.0 & 81.6 \\
\hline $\mathrm{EE}-4$ & 0.0 & 21.1 & 5.3 & 0.0 & 73.7 \\
\hline EI-1 & 0.0 & 13.2 & 10.5 & 68.4 & 7.9 \\
\hline EI-2 & 2.6 & 15.8 & 2.6 & 57.9 & 21.1 \\
\hline EI-3 & 0.0 & 13.2 & 13.2 & 55.3 & 18.4 \\
\hline EI-4 & 0.0 & 26.3 & 0.0 & 23.7 & 50.0 \\
\hline EO-1 & 2.6 & 7.9 & 7.9 & 26.3 & 55.3 \\
\hline EO-2 & 0.0 & 5.3 & 15.8 & 15.8 & 63.2 \\
\hline EO-3 & 0.0 & 7.9 & 0.0 & 47.4 & 44.7 \\
\hline EO-4 & 0.0 & 5.3 & 5.3 & 7.9 & 81.6 \\
\hline IA-1 & 0.0 & 0.0 & 63.2 & 13.2 & 23.7 \\
\hline IA-2 & 2.6 & 5.3 & 44.7 & 23.7 & 23.7 \\
\hline IA-3 & 2.6 & 0.0 & 84.2 & 7.9 & 5.3 \\
\hline $\mathrm{IA}-4$ & 0.0 & 2.6 & 89.5 & 0.0 & 7.9 \\
\hline IE-1 & 2.6 & 13.2 & 2.6 & 13.2 & 68.4 \\
\hline IE-2 & 0.0 & 44.7 & 0.0 & 36.8 & 18.4 \\
\hline IE-3 & 0.0 & 36.8 & 2.6 & 21.1 & 39.5 \\
\hline IE-4 & 0.0 & 31.6 & 0.0 & 34.2 & 34.2 \\
\hline III-1 & 0.0 & 5.3 & 31.6 & 7.9 & 55.3 \\
\hline II -2 & 2.6 & 5.3 & 50.0 & 5.3 & 36.8 \\
\hline II-3 & 0.0 & 5.3 & 28.9 & 2.6 & 63.2 \\
\hline II -4 & 0.0 & 0.0 & 39.5 & 2.6 & 57.9 \\
\hline $10-1$ & 5.3 & 2.6 & 0.0 & 39.5 & 52.6 \\
\hline $10-2$ & 2.6 & 7.9 & 7.9 & 42.1 & 39.5 \\
\hline $10-3$ & 0.0 & 2.6 & 18.4 & 34.2 & 44.7 \\
\hline $10-4$ & 0.0 & 2.6 & 7.9 & 44.7 & 44.7 \\
\hline OA-1 & 0.0 & 5.3 & 5.3 & 60.5 & 28.9 \\
\hline $\mathrm{OA}-2$ & 0.0 & 7.9 & 13.2 & 63.2 & 15.8 \\
\hline $\mathrm{OA}-3$ & 0.0 & 2.6 & 18.4 & 78.9 & 0.0 \\
\hline $\mathrm{OA}^{-4}$ & 2.6 & 5.3 & 13.2 & 50.0 & 28.9 \\
\hline $\mathrm{OE}-1$ & 2.6 & 13.2 & 0.0 & 2.6 & 81.6 \\
\hline OE-2 & 0.0 & 5.3 & 10.5 & 26.3 & 57.9 \\
\hline $\mathrm{OE}-3$ & 0.0 & 15.8 & 7.9 & 23.7 & 52.6 \\
\hline $\mathrm{OE}-4$ & 0.0 & 10.5 & 26.3 & 5.3 & 57.9 \\
\hline OI-1 & 5.3 & 0.0 & 7.9 & 28.9 & 57.9 \\
\hline $\mathrm{OI}-2$ & 0.0 & 5.3 & 13.2 & 47.4 & 34.2 \\
\hline OI-3 & 2.6 & 2.6 & 15.8 & 31.6 & 47.4 \\
\hline $\mathrm{OI}-4$ & 5.3 & 2.6 & 7.9 & 26.3 & 57.9 \\
\hline OO-1 & 2.6 & 2.6 & 13.2 & 21.1 & 60.5 \\
\hline OO-2 & 0.0 & 10.5 & 28.9 & 13.2 & 47.4 \\
\hline OO-3 & 2.6 & 0.0 & 10.5 & 21.1 & 65.8 \\
\hline $\mathrm{OO}-4$ & 2.6 & 0.0 & 7.9 & 23.7 & 65.8 \\
\hline
\end{tabular}

Note-Numbers indicate percentage of total responses to each syllogism. $\quad{ }^{*}$ refers to "no valid conclusion." number of correct responses have been demonstrated for premise combinations EI, IE, and AE for Sample 1 and for premise combinations EI and IE for Sample 2, with the difference on premise combination EA approaching significance. Of the 24 relevant syllogisms for the two samples combined, figure effects have been demonstrated for 18 , with 2 more approaching significance.

These data also support the explanation of figure effects in terms of backward processing. Thus, figure effects on the occurrence of the predicted error have been demonstrated for premise combinations EI, IE, $\mathrm{EA}$, and $\mathrm{AE}$ for Sample 1 and for premise combinations EI, IE, and EA for Sample 2. Of the 24 relevant syllogisms for the two samples combined, figure effects have been demonstrated for 22 .

Thus, the decrement in the number of correct responses across figures is paralleled by an increase in the occurrence of the error that is predicted on the basis of backward processing. The more strongly the direction of the information in the premises promotes backward processing, the greater the number of errors corresponding to the valid conclusion for backward processing at the expense of the correct conclusion, based upon forward processing that is required of the subject.

Additional strong support for the explanation in terms of backward processing is provided by the analyses of those premise combinations for which both forward and backward processing lead to the same conclusions. In all of these instances, there are no significant differences between figures. Thus, figure differences only occur in those instances where the conclusions derived from forward and backward processing are discrepant.

This demonstration of the effects of backward processing provides evidence for a new kind of illicit conversion to supplement the principle of conversion of premises proposed by Chapman and Chapman (1959). In the present instance, the subject is erroneously converting conclusions. Thus, the conclusion "Some $\mathbf{P}$ are not S" is erroneously converted to "Some $S$ are not P." Similarly, the conclusion that no propositional assertion can be deduced from $\mathbf{P}$ to $\mathbf{S}$ is erroneously converted into the conclusion that no propositional assertion can be deduced from $\mathrm{S}$ to $\mathrm{P}$.

It is possible to reformulate these error tendencies in terms of a more general proposition. In all of the instances in which a subject erroneously converts a proposition, the subject is assuming a symmetrical relation between the classes represented by the terms of the syllogism. Such a symmetrical relation is less complex than an asymmetrical relation in which the form of the relation proceeding in the forward direction is different from the form of the relation proceeding in the backward direction. Some evidence that this preference for symmetrical relations is a general tendency on reasoning tasks is provided by studies 
of conditional reasoning (Wason \& Johnson-Laird, 1972), in which subjects often erroneously assume that the proposition, "If $p$ then q" implies both "If q then $p$ " and "If not $p$ then not $q . "$

The present study also replicates the figure effect based upon all 19 valid syllogisms reported by Erickson (1974). However, as noted above, the comparison across figures for these syllogisms is confounded due to the unequal distribution of conversion syllogisms across figures. In addition, the difference is partially attributable to the difference across figures on premise combination EI, which has been demonstrated in the present paper as a function of backward processing. When the four conversion and four EI syllogisms are removed from the set of valid syllogisms, there is no significant figure effect for the remaining 11 syllogisms in either sample.

The data of the present study do not support the mediation paradigm analogy proposed by Frase (1968). Predictions from the mediation paradigm predict figure effects on a broader range of syllogisms than those predicted by the backward processing explanation. Yet, the only significant differences occur where predicted on the basis of backward processing. Furthermore, the backward processing explanation successfully predicts the specific errors which will occur, while the mediation paradigm analogy does not. Thus, the present study supports the greater utility of an information processing approach to syllogistic reasoning, as opposed to an associative approach.

The figure differences demonstrated in this study are not predicted by any of the general models that have been proposed to account for subject performance on the syllogistic task. The atmosphere effect explanation (Woodworth \& Sells, 1935) bases its predictions on the kinds of propositions (A, E, I, or $O$ ) that occur in the major and minor premises. Since premise combinations remain the same across all four figures, no figure effects are predicted. Chapman and Chapman (1959) confine their attention to invalid syllogisms, which they divide into two subsets, conversion syllogisms and probabilistic inference syllogisms. However, in both instances, predictions for a given premise combination remain the same across figures.

More recently, Erickson (1974) has presented a set of quantitative models for the syllogistic task. For the comparisons under consideration in this paper, these models do not make differential figure predictions for premise combinations IE, EA, and AE. Indeed, in all of these instances, the data from the various figures are combined and are not reported individually. The only premise combination relevant to this paper for which Erickson (1974) predicts a figure effect is EI. Here, the models predict that performance will be better on Figures 1 and 2 than on Figures 3 and 4, and that this decrement will be paralleled by an increase in $E$ errors in Figures 3 and 4 . Unfortunately, the data reported by Erickson (1974) do not support these predictions. In fact, the percentage correct in these data is slightly higher in Figures 3 and 4 , while the percentage of $E$ errors in these figures is slightly lower. While these data are discrepant with the predictions of Erickson's (1974) models, they do not present any difficulty for the backward processing explanation. Erickson's (1974) procedure did not provide subjects with the alternative of a nonpropositional conclusion, and no data are reported for this category. Since the backward processing explanation predicts that the decrement in percentage correct across figures for premise combination EI results from an increase in nonpropositional conclusions, no figure effect would be expected when this option is ruled out by the procedure. Thus, Erickson's (1974) data provide additional, indirect support for the backward processing explanation.

Revlis (1975b) has presented a model which combines the principle of conversion proposed by Chapman and Chapman (1959) with the principle of feature selection based upon the atmosphere explanation of Woodworth and Sells (1935). However, again, for the comparisons under consideration in this paper, no figure differences are predicted. For valid premise combination EI, the model predicts correct performance in all four figures. Thus, the model fails to predict the substantial frequency of nonpropositional conclusions demonstrated in this paper and also fails to predict the significant figure differences. For valid premise combination $E A$ in Figures 3 and 4 , the model correctly predicts the E error based upon conversion, which is the dominant error in both figures, but fails to predict the significant difference between figures in nonpropositional conclusions. For invalid premise combination $\mathrm{AE}$ in Figures 1 and 3 , the model correctly predicts the $E$ error based upon conversion, which is the dominant error in both figures, but fails to predict the significant differences between figures in both the number correct and the number of $\mathrm{O}$ errors. Finally, for invalid premise combination IE, the model correctly predicts the occurrence of 0 errors, as predicted by the backward processing explanation. However, for Revlis (1975b), this error is due to feature selection based upon the atmosphere of the premises. Since the atmosphere is the same for all four figures, no figure differences are predicted. Since the present data clearly demonstrate figure differences in this error, the backward processing explanation appears to provide a better account of subject performance than does the principle of feature selection. It may also be noted that there is other evidence (e.g., Ceraso \& Provitera, 1971; Dickstein, 1975) against the utility of a feature selection or atmosphere explanation of subject performance.

In summary, none of the existing models of syllogistic reasoning predicts the occurrence of the figure 
effects demonstrated in this paper, and thus, none can be regarded as a fully comprehensive account of subject performance on this task. Rather, it appears that any complete model will have to incorporate the principle of backward processing as one of its components.

The present study provides partial support for the prediction that performance would be better on valid premise combination EI than on invalid premise combination IE, based upon the assumption (Revlis, $1975 b$ ) that subjects have a bias against nonpropositional conclusions. This prediction was confirmed for Sample 1 but not for Sample 2. In the second sample, while there was no main effect of validity, there was a significant interaction between figure and validity for correct responses. This interaction is difficult to explain, since it was not accompanied by a significant interaction for the predicted errors.

Finally, it may be noted that the present study used a multiple choice format, in which the subject was provided with four propositional conclusions that always went from $S$ to $P$ as well as one nonpropositional conclusion to choose from. Wason and Johnson-Laird (1972) have noted that such a task involves the evaluation of conclusions by the subject, rather than the more active construction of conclusions. They have suggested the use of a task in which only the premises are provided and the subject is free to write any conclusion. Such a task would seem to have special utility in future research on the effects of figure, since it would provide more direct evidence for the occurrence of backward processing than is possible with the multiple choice format.

\section{REFERENCES}

Bruning. I. \& Kintz. 8. Computational handbook of statistics. Glenview. 1ll: Scott Foresman, 1968.

Ceraso, J.. \& Provitera, A. Sources of error in syllogistic reasoning. Cognitive Psychology. 1971, 2. 400-410.

Chapman, L.. \& Chapman, J. Atmosphere effect reexamined. Joumal of Experimental Psychology. 1959, 58, 220-22b.

Dickstein. L. S. Effects of instructions and premise order on errors in syllogistic reasoning. Journal of Experimental
Psycholigy: Human Leaming and Memory, 1975, 1. 376-384.

Dickstein, L. S. Differential difficulty of categorical syllogisms. Bulletin of the Psychonomic Society, 1976. 8. $330-332$

ERICKSON, J. R. A set analysis theory of behavior in formal syllogistic reasuning tasks. In R. L. Solso (Ed.), Theories in cognitive psichology: The Lovola symposium. Hillsdale. N.J: Lawrence Erlbaum, 1974.

Frase. L. T. Associative factors in syllogistic reasoning. Journal of Experimental Psychology, 1968, 76, 407-412.

Kintsch, W., \& Monk, D. Storage of complex information in memory: Some implications of the speed with which inferences can be made. Journal of Experimental Psychology, 1972. 94, 25-32.

Revis, R. Two models of syllogistic reasoning: Feature selection and conversion. Journal of Verbal Learning and Verbal Behavior, 1975, 14, 180-195. (a)

Revlis, R. Syllogistic reasoning: Logical deductions from a complex data base. In R. J. Falmagne (Ed.), Reasoning: Representation and process. Hillsdale, N.J: Lawrence Erlbaum, 1975. (b)

ROBERGE, J. A reexamination of the interpretation of errors in formal syllogistic reasoning. Psychonomic Science, 1970, 19, 331-333.

ROBERGE, J. Further examination of mediated associations in deductive reasoning. Journal of Experimental Psychology', 1971, 87, 127-129.

Wason, P. C., \& JoHNSON-LaIRD, P. N. Psychology of reasoning: Simuture and content. Cambridge: Harvard University Press, 1972.

WOODWORTH. R.. \& SELLS. S. An atmosphere effect in formal syllogistic reasoning. Journal of Experimental Psychology, 1935. 18. 451-460.

\section{NOTE}

1. The number of correct responses and the number of predicted errors across figures are not independent analyses, since the number of errors is constrained by the number correct. Both analyses are presented to demonstrate that figure differences in performance are reflected in the specific errors predicted by the hypothesis of backward processing and do not simply represent an increase in incorrect responses of all types.

(Received for publication July 14,1977 ; revision accepted September 22, 1977.) 